\title{
Evaluación de la fase de screening auditivo en menores con factores de riesgo
}

\author{
Evaluation of the auditive screening stage \\ in minors with risk factors
}

\author{
Cristián Godoy $\mathrm{B}^{1}$, Loretho Bustamante $\mathrm{M}^{1}$.
}

\begin{abstract}
RESUMEN
Se analizan los resultados obtenidos en la primera fase del programa de Detección Precoz de la Sordera en el Hospital Sótero del Río durante el año 2004. Se estudia un total 244 casos, evaluados en el Servicio de Corrinolaringología (ORL), en cuanto a los resultados obtenidos de la primera evaluación con emisiones otoacústicas transientes (EOAT) automáticas, en el Servicio de Neonatología, examen impedanciométrico y emisiones otoacústicas por producto de distorsión (EOAPD) clínica Además, se evalúa el tiempo promedio que transcurre entre el screning tomado en la maternidad y el primer examen de emisiones otoacústicas (EOA) clínico, en 144 casos. Como resultados se obtiene que $d$ $22,8 \%$ de los menores evaluados en neonatología son derivados a rescreening y $6 \%$ va a estudio audiológico diagnóstico. En cuanto a la eficiencia del sistema se desprende un promedio de 14 días entre el screening de la maternidad y la primera EOA clínica

Palabras clave: Screening auditivo, Emisiones Otoacústicas.
\end{abstract}

\section{SUMMARY}

The results obtained in the first stage of the Early Detection of Deafness at Dr. Sótero del Rio Hospital during year 2004 are analyzed. A total of 224 cases are surveyed, evaluated at the ORL Service, in what refers to results obtained from the first evaluation with automatic transient otoacoustic emissions (EOAT), at the Neonatology Service, impedance exam and otoacoustic emissions as a result of clinical distortion (EOAPD). Also, the average time elapsed between the screening made in the maternity ward and the first clinical exam of otoacoustic emissions (EOA) is evaluated in 144 cases. The result obtained is that $22,8 \%$ of the minors evaluated in neonatology are derived for re screening and $6 \%$ goes to diagnostic hearing study. In connection with the efficiency of the system, an average of 14 days elapses between the screening in maternity ward and the first clinical EOA

Key words: Auditive screening, Otoacoustic Emissions.

\footnotetext{
${ }^{1}$ Fonoaudiólogos del Servicio de Aorrinolaringología del Hospital Dr. Sótero del Río.
} 


\section{INTRODUCCIÓN}

日 objetivo más importante de la detección precoz de la sordera es lograr una implementación auditiva adecuada, en un tiempo crítico, que permita un desarrollo evolutivo y comunicativo apropiado ${ }^{1}$.

Estudios muestran que los niños con hipoacusia sensorioneural (HSN) que son implementados tempranamente, tienen una habilidad lingüística mejor que los que son intervenidos tardíamente?

La HSN está presente en 2 de cada 1.000 recién nacidos vivos. De éstos, el $61 \%$ proviene de las unidades de cuidados intensivos ${ }^{3}$. Los factores de riesgo para presentar HSN son: peso al nacer menor de 1.500 gramos, hiperbilirrubinemia, asistencia ventilatoria por más de 5 días, anomalías craneofaciales, TORCH, medicamentos ototóxicos, sufrimiento fetal, asfixia neonatal y antecedentes familiares de hipoacusia sensorioneural ${ }^{4}$.

日 Servicio de Otorrinolaringología del Hospital Dr. Sótero del Río presentó el año 2002 la experiencia inicial en el diagnóstico de hipoacusia neonatal, demostrando que es posible organizar un sistema eficiente y coordinado para poner en marcha este programa. Ese estudio demostró que se llegó a diagnóstico en el $97 \%$ de los casos ${ }^{5}$.

Nuestro interés ahora está centrado en analizar la primera etapa de este programa, es decir la etapa de screening.

Para realizar screening auditivo se han descrito distintos métodos. Se utilizan en este caso emisiones otoacústicas (EOA), las que pueden ser de tipo transientes o por producto de distorsión.

Las EOAT muestran la respuesta de las células ciliadas externas $(\mathrm{CE})$ en un amplio rango de frecuencias, frente a un estímulo clic. Las EOAPD muestran la respuesta de las $\mathrm{CEE}$ para una frecuencia determinada.

Estos métodos presentan una especificidad del 83\%-92\% y una sensibilidad del $84 \%-86 \%$. Destaca su sencillez, fiabilidad y rapide $z^{5}$.

日 propósito de este trabajo es comunicar la experiencia del Servicio de Otorrinolaringología del Hospital Sótero del Río, respecto a la fase de screening del programa de detección precoz de la sordera durante el año 2004, evaluando el tiempo que transcurre entre la primera y segunda evaluación y los resultados del examen impedanciométrico y de EOA.

\section{MATERIAL Y MÉTODO}

Se realiza un estudio retrospectivo de los menores atendidos en el servicio de ORL del Hospital Sótero del Río, incluidos en el programa de detección precoz de sordera entre enero y diciembre de 2004.

Se analizan los datos del libro de evaluación auditiva de neonatología, donde se consignan los resultados obtenidos por las matronas, en la evaluación con EOAT automáticas y los resultados consignados en la tabla de registro en planilla Excel, realizada por los fonoaudiólogos en el laboratorio de audiología. Este registro tiene por objetivo llevar un control de los pacientes que son diagnosticados y su seguimiento.

La etapa de screening comienza en el Servicio de Neonatología donde se realiza un examen auditivo, con EOAT automáticas, con el equipo Madsen Eccho Scren, previo al alta Aquellos niños que no pasan este examen ya sea unilateral o bilateral, son derivados al servicio de otorrinolaringología del mismo hospital. En el Servicio de ORL el estudio auditivo es realizado por los fonoaudiólogos, los que aplican un examen timpanométrico (Impedanciómetro Madsen Zodiac 901) con el objetivo de descartar presencia de líquido en el oído medio y luego un re-screening con EOAPD con el equipo Madsen Capella La elección de esta modalidad se debe a que el examen es más rápido y específico en relación a las frecuencias que deseamos estudiar, en este caso 2.000, 4.000 y 8.000 $\mapsto z$ Aquellos niños que no pasan este examen en forma unilateral o bilateral, pasarán a la segunda fase del programa, donde se efectuará el diagnóstico de sordera a través del estudio con impedanciometría, Potenciales Evocados Auditivos de Tronco (PEAT) y observación de conducta Los niños que presentan exámenes sin alteración son dados de alta con instrucciones de observación a los padres. 
La información obtenida de los registros del Servicio de Neonatología y de ORL, fue analizada utilizando métodos estadísticos descriptivos, y sus resultados se muestran a continuación.

\section{RESULTADOS}

Durante el año 2004 nacieron y fueron atendidos en la Unidad de Neonatología 1.272 menores, de los cuales 799 fueron evaluados con screening por las matronas $(62,8 \%)$.

Los niños que no pasaron el primer examen de EOAT fueron 182 (22,8\%), y fueron derivados al Servicio de Otorrinolaringología para realizar el re screening.

En el laboratorio de audiología se evaluaron 144 niños de los 182 que no pasaron el primer examen en neonatología, perdiéndose 38 recién nacidos $(20,9 \%)$ y 100 menores que no fueron evaluados durante su estadía en neonatología. Por lo tanto se analizaron 244 estudios de screening en nuestra unidad.

Ladistribución por sexo de la muestra corresponde a 150 hombres $(61,4 \%)$ y 94 mujeres $(38,5 \%)$.
Esta reevaluación teóricamente debe realizarse en los 15 días posteriores al alta neonatológica. La latencia promedio de la muestra correspondió a 19,7 días, con un rango de 1 a 96 días.

Se realizó timpanometría en 214 menores, dos de los cuales presentaban agenesia unilateral de oído por lo que se obtienen 426 impedanciometrías, de éstas, 399 presentan curva A (93,6\%), 14 oídos presentan curva $B(3,3 \%)$ y 13 oídos presentan curva $C(3,1 \%)$. Los resultados se muestran en la Figura 1.

Del examen de EOAPD realizado en 244 menores se obtuvieron los siguientes resultados: 177 menores pasan la prueba $(72,5 \%), 45$ niños no pasan en ambos oídos (18,4\%), 8 niños no pasan en el OD (3.3\%) y 14 niños no pasan en el O $(5,7 \%)$ (Fgura 2). Por lo tanto pasan a estudio de sordera unilateral o bilateral 67 niños (27,4\%).

De los 144 menores evaluados en neonatología y que no pasan el examen y son reevaluados, 96 niños (67\%) pasa el re-screning y 48 niños (33\%) no pasa el segundo examen (sea uni o bilateral) y van a estudio diagnóstico. Del total de 799 niños estudiados en neonatología inicialmente, sólo el $6 \%$ va a estudio de sordera.
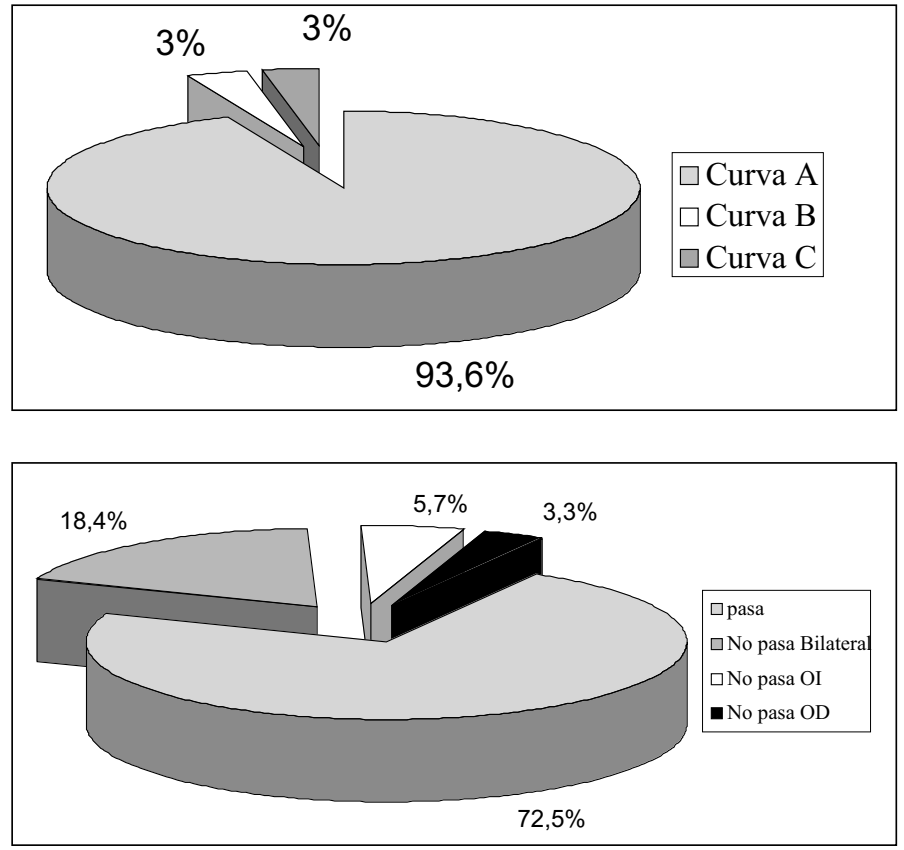

Figura 1. Resultados impedanciometría.

Figura 2. Resultados EOAPD. 
日 estudio diagnóstico contempla timpanograma, potenciales evocados auditivos de tronco cerebral y observación de conducta auditiva Quando los resultados están alterados o dudosos son presentados al médico otorrinolaringólogo.

\section{CONCLUSIONES Y DISCUSIÓN}

En este trabajo se obtiene que el $22,8 \%$ de los menores evaluados en neonatología son derivados a re-screening. De este grupo el $6 \%$ va a estudio audiológico diagnóstico. La cobertura del screening alcanza al $62,8 \%$.

Estos resultados concuerdan con publicaciones internacionales, donde el porcentaje de menores que refiere el primer screening varía entre un $27 \%-34,61 \%$ y el porcentaje de menores que, después del segundo screening, va a estudio audiológico diagnóstico, varía entre 7,14\%$12 \% 6-7$.

Entre las observaciones realizadas durante el proceso de recolección de datos, consideramos que podrían obtenerse mejores resultados con la existencia de un registro eficiente, único y coordinado entre los servicios de neonatología y otorrinolaringología. Además se propone que sea un profesional quien se encargue de la coordinación entre ambos servicios, de los profesionales involucrados y el seguimiento de cada paciente.

También se observa que la carencia de equipos de repuesto en los servicios provocó que, en 100 niños no se realizara el primer screening y que en 30 menores no se realizara timpanometría. Esto nos lleva a sugerir que el sistema debe considerar la reposición de equipos en un breve periodo de tiempo.

Por último, creemos que la implementación de la primera fase del programa de detección precoz de sordera del Hospital Sótero del Río, aunque puede ser mejorada, cumple con los criterios recomendados en la literatura especializada.

\section{BIBLIOGRAFÍA}

1. GONZÁLEz L. Detección precoz de la hipoacusia en recién nacidos prematuros en el Hospital Sótero del Río. Tesis para optar al título de fonoaudiólogo. Universidad de Valparaíso. 2001.

2. Bailey H, BOWER C, KRISHNASWAMy J, COATES H. Newborn Hearing Screening in Western Australia. MJA2002; 177: 180-5.

3. DALZZL L ET AL. The New Cork State Universal Newborn hearing screening demostration project: Ages of hearing loss identification, hearing aid fitting and enrollment in early intervention. Ear Hear 2000; 21: 118-30.

4. Year 2000 Position Statement: Principles and guidelines for early hearing detection and intervention programs. Joint Committee on Infant Hearing, American Academy of Audiology, American Academy of Pediatrics, American Speech-Languaje-Hearing Association, and Directors of Speech and Hearing Programs in State Health and Welfare Agencies. 2000.

5. INIIGUEZ R, CEVOT, FERNÁNDEZ F, GODOY C, IÑIGUEZ R. Detección Precoz de Pérdida Auditiva en Niños con Factores de Riesgo. Rev Otorrinolaringol Or Cabeza Ouello 2004; 64: 99-104.

6. Zhao PJ, Xu ZM, Wu SH, Jin CH, Yu H, Shen P, Li J, Wang WC, Shen XM. Hearing screening for high-risk newborns. Zhonghua Yi Xue Za Zhi 2003; 25; 83(4): 285-8.

7. $\mathrm{Xu} Z \mathrm{M}, \mathrm{Li} J$, Hu TZ, Sun JH, Shen XM. Sensitivity of distortion product otoacustic emissions and auditory brain-stem response in neonatal hearing screening, a comparative study. Zhonghua Yi Xue Za Zhi 2003; 25; 83(4): 278-80. 